\title{
Revised vs original QUICKI index during diet + rosiglitazone treatment in obese subjects
}

\author{
A Brunani ${ }^{1}$, A Caumo ${ }^{2}$, S Graci ${ }^{1}$, C Margarini ${ }^{1}$, GC Viberti ${ }^{3}$, A Liuzzi ${ }^{1}$
}

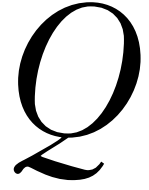

UICKI is an index of insulin sensitivity based on the determination of glucose and insulin concentrations in the postabsorptive state [1]. It has been shown to be well correlated with insulin sensitivity across the entire spectum of glucose tolerance, but its performance is less satisfactory in normal subjects [1]. To overcome this drawback, QUICKI has been subsequently revised with the incorporation of plasma non esterifed fatty acids (NEFA) concentrations [2] in the formula. The revised QUICKI performs better than the original QUICKI to measure of insulin sensitivity in normal subjects and in non obese subjects with mild insulin resistance. However, in obese subjects, Bastard et al. [3] have recently shown that the significant increase of insulin sensitivity induced by weight loss is reliably detected by QUICKI, but not by the revised QUICKI.

Aim of this study was to determine whether this effect was present when studying obese subjects undergoing a therapy with diet and rosiglitazone.

We treated 13 non diabetic obese subjects $(5$ men, 8 women; age $19-51 \mathrm{yrs}$, BMI $42,21 \mathrm{r} 2,91 \mathrm{~kg} / \mathrm{m}^{2}$ ) with hypocaloric diet $(18 \%$ proteins, $58 \%$ carbohydrates and $24 \%$ lipids) setting on $75 \%$ of basal metabolic rate (BMR)(range 1400-1600 kcal per day) and rosiglitazone (4 mg u 2/die) for 6 months. This drug increases insulin sensitivity through an agonist effect on peroxisome proliferator-activated receptor-gamma (PPARJ) and enhances the ability of the body to utilize NEFA during fasting [4]. The overall result is an improvement of both glucose (increased peripheral glucose uptake and E-cell function) and lipid metabolism (reduced NEFA levels) [5].

Several metabolic parameters were measured in our subjects in basal conditions and after weight loss: body mass index (BMI), fat mass (FAT), free fat mass (FFM), basal metabolic rate (BMR), glucose, insulin and NEFA plasma levels, QUIKI and revised QUICKI. Plasma glucose was measured enzymatically using Roche kit and reagent on a Hitachi analyzer; plasma insulin was measured by immunometric assay (IMMULITE ${ }^{\circledR}$ 2000, DPC, Los Angeles,

\footnotetext{
${ }^{1}$ Department of Internal Medicine, Ospedale San Giuseppe, IRCCS, Istituto Auxologico Italiano, Verbania, Italy

${ }^{2}$ Metabolic and Nutrition Unit, Instituto Scientifico H San Raffaele, Milan, Italy

${ }^{3}$ Department of Diabetes and Endocrinology, GKT School of Medicine,

Guy's Hospital, King's College London, London, United Kingdom.
}

USA). A blood sample for NEFA was collected into EDTA tubes kept on ice and plasma separated off within four hours of collection. The samples were stored at -20oc until analysis performed using enzymatic colorimetric method (Wako Chemicals, Richmond, VA, USA). CV intra- and inter-assay were $0.7 \%$ and $1.8 \%$, respectively.

All data are presented as mean $r$ s.e. Student's two-tailed paired t-test with Bonferroni adjustment was used to compare the metabolic parameters before and after treatment. A $\mathrm{p}$ value $<.05$ was considered statistically significant.

At the end of the study period we obtained a significant weight loss (Tab I) accompanied by a significant reduction in FAT mass. Insulin and glucose levels decreased significantly, which determined a significant increase in the QUICKI index. The NEFA levels markedly decreased in 9 out of 12 patients ( $-31 \%$ on average), but markedly increased $(+52 \%$ on average $)$ in 3 patients, the overall reduction resulting not significant. As a result, in these 3 patients the revised QUICKI increased much less than the original QUICKI. Nevertheless. when the 12 patients were considered althogether, the increase of the revised QUICKI reached the statistical significance.

Our data show that in obese subjects the reduction in weight loss induced by diet and rosiglitazone is accompanied by a significant reduction in glucose and insulin levels. As a result, the QUICKI index increases, suggesting a significant enhancement of insulin sensitivity in these patients. This is in keeping with the well-established notion that weight loss mitigates insulin resistance in obese patients [6] and with a recent report by Virtanen et al. [7] showing an improvement in insulin sensitivity in diabetic patients receiving rosiglitazone. NEFA levels remained unaltered on average, but scrutiny of the individual data showed that all subjects responded to the therapy with a marked change in their NEFA levels: in 9 out 12 subject NEFA levels decreased, whereas in the remaining 3 subjects NEFA levels increased. In support of this finding of heterogeneous behaviour of NEFA levels, there is the evidence of no significant correlation between NEFA concentrations and the expression proteins involved

Address correspondence and reprint requests to:

A Brunani. Istituto Auxologico Italiano, Ospedale San Giuseppe, Piancavallo, 28900 Verbania, Italy. brunani@auxologico.it

Received: July 19th, 2004 
Table I

Anthropometric and laboratory data in obese patients $(n=13)$.

\begin{tabular}{|c|c|c|}
\hline & Baseline & $\begin{array}{l}\text { Weight loss + } \\
\text { rosiglitazone }\end{array}$ \\
\hline BMI $\left(\mathrm{kg} / \mathrm{m}^{2}\right)$ & 42.21 r 0.81 & 38.19 r $0.87^{* *}$ \\
\hline FAT mass kg & 52.21 r 2.33 & 43.76 r 2.25* \\
\hline BMR (kcal/die) & 1988 r 126 & 1997 r 137 \\
\hline Blood glucose (mg/dl) & 83.77 r 1.77 & 78.85 r 1.8 * \\
\hline Insulin (PU/ml) & 13.56 r 1.50 & 7.97 r 0.66 * \\
\hline NEFA (mmol/l) & 0.70 r 0.10 & 0.59 r 0.20 \\
\hline QUICKI index & 0.332 r 0.056 & $0.361 \mathrm{r} 0.055^{*}$ \\
\hline Revised QUICKI index & 0.351 r 0.023 & 0.399 r 0.036 * \\
\hline
\end{tabular}

(means $r$ s.e.)

BMI: body mass index; BMR: basal metabolic rate; NEFA: non

esterified fatty acids; QUICKI index: 1/[log (glucose) + log

(insulin)]; Revised QUICKI index: 1/[log (glucose) + log (insu-

lin) $+\log ($ NEFA) $]$.

${ }^{*} p<0.005$ vs baseline ${ }^{* *} p<0.001$ vs baseline

in fatty acid metabolism in adipose tissue [8]. The practical consequence of this mixed behavior on NEFA levels was that the revised QUICKI index changed little at the end of the study, and was less capable than the original QUICKI index to reveal the improvement in insulin sensitivity experienced by the subjects.

The present data confirm the pattern of the results published by Bastard et al. [3]. They suggest that the original QUICKI is more effective than the revised QUICKI index in monitoring the improvement in insulin sensitivity induced by a combined therapy with diet and rosiglitazone. Our finding that $25 \%$ of our patients show increased NEFA levels at the end of the study suggests a possible explanation for this outcome. We speculate that the combined diet and rosiglitazone therapy is able to induce an increased lypolisys that in turn produces a transient elevation of NEFA concentration (corresponding to the elimina- tion of stored lipids in fat). This initial phase is followed by a reduction of NEFA concentration, and finally by the attainment of a steady-state NEFA level (presumably lower than the initial one) reflecting the new hormonal/metabolic milieu induced by therapy. Since the dynamics of this process may require a long time and may vary considerably among subjects, it may happen that NEFA are measured at different stages of this process, and often when they are still far from their final steady-state level. Under such conditions, NEFA levels are unreliable indicators of insulin action, which leads to a suboptimal performance of the revised QUICKI index.

\section{References}

1. Katz A, Nambi SS, Mather K, et al. Quantitative insulin sensitivity check index: a simple, accurate method for assessing insulin sensitivity in human. J Clin Endocrinol Metab, 2000, 85, 2402-10.

2. Perseghin G, Caumo A, Caloni M, Testolin G, Luzi L. Incorporation of the fasting plasma FFA concentration into QUICKI improves its association with insulin sensitivity in nonobese individuals. J Clin Endocrinol Metab, 2001, 86, 4776-81.

3. Bastard JP, Jan V, Maachi M, et al. Incorporation of non esterified fatty acids into QUICKI is not relevant in obese subjects during diet induced weight loss. Diabetes Metab, 2002, 28, 333-4.

4. Oakes ND, Thalén PG, Jacinto SM, Ljung B. Thiazolidinediones increase plasma-adipose tissue FFA exchange capacity and enhance insulin-mediated control of systemic FFA availability. Diabetes, 2001, 50, 1158-65.

5. Mayerson AB, Hundal RS, Dufour S, et al. The effects of rosiglitazone on insulin sensitivity, lipolysis, and hepatic and skeletal muscle triglyceride content in patient with type 2 diabetes. Diabetes, 2002, 51, 797-802.

6. Costacou T, Mayer-Davis EJ. Nutrition and prevention of type 2 diabetes. Annu Rev Nutr, 2003, 23, 147-70.

7. Virtanen KA, Hallsten K, Parkkola R, et al. Differential effects of rosiglitazone and metformin on adipose tissue distribution and glucose uptake in type 2 diabetic subjects. Diabetes , 2003, 52, 283-90.

8. Fisher RM, Hoffstedt J, Hotamisligil GS, Thorne A, Rydén M. Effects of obesity and weight loss on the expression of proteins involved in fatty acid metabolism in human adipose tissue. Int J Obes Relat Metab Disord, 2002, 26, 1379-85. 